Submitted Aug 1, 2007; Accepted Sep 19, 2007 for Publication in the Astrophysical Journal

Preprint typeset using LATEX style emulateapj v. 08/22/09

\title{
XMM-NEWTON OBSERVATION OF THE CLUSTER ZW 1305.4+2941 IN THE FIELD SA 57
}

\author{
Fabio Gastaldello ${ }^{1,2}$, Dario Trevese ${ }^{3}$, Fausto Vagnetti ${ }^{4}$, Roberto Fusco-Femiano ${ }^{5}$ \\ Submitted Aug 1, 2007; Accepted Sep 19, 2007 for publication in the Astrophysical Journal
}

\begin{abstract}
We report the details of an XMM observation of the cluster of galaxies Zw $1305.4+2941$ at the intermediate redshift of $z=0.241$, increasing the small number of interesting X-ray constraints on properties of $\sim 3 \mathrm{keV}$ systems above $z=0.1$. Based on the $\sim 45 \mathrm{ks} X M M$ observation, we find that within a radius of $228 \mathrm{kpc}$ the cluster has an unabsorbed X-ray flux of $f_{\mathrm{X}}=(2.07 \pm 0.06) \times 10^{-13} \mathrm{ergs}^{-2} \mathrm{sm}^{-1}$, a temperature of $k T=3.17 \pm 0.19 \mathrm{keV}$, in good agreement with the previous $R O S A T$ determination, and an abundance of $0.93_{-0.29}^{+0.24} Z_{\odot}$. Within $r_{500}=723 \pm 16 \mathrm{kpc}$ the rest-frame bolometric X-ray luminosity is $L_{\mathrm{X}}\left(r_{500}\right)=(1.25 \pm 0.16) \times 10^{44} h_{70}^{-2} \mathrm{ergs} \mathrm{s}^{-1}$. The cluster obeys the scaling relations for $L_{\mathrm{X}}, T$ and the velocity dispersion $\sigma_{v}$ derived at intermediate redshift for $k T \lesssim 4 \mathrm{keV}$, for which we provide new fits for all literature objects. The mass derived from an isothermal NFW model fit is, $M_{\text {vir }}=2.77 \pm 0.21 \times 10^{14} \mathrm{M}_{\odot}$, with a concentration parameter, $c=7.9 \pm 0.5$.

Subject headings: galaxies: clusters: general — X-rays: general
\end{abstract}

\section{INTRODUCTION}

The current generation of X-ray observatories, Chan$d r a$ and $X M M$, is considerably extending the maximum redshift to which $\mathrm{X}$-ray clusters can be identified and analyzed. In fact the number of massive clusters detected at $z>1$ is rapidly growing thanks to the unprecedented $X M M$ sensitivity (e.g, Mullis et al. 2005; Stanford et al. 2006; Bremer et al. 2006). On the other hand they are also extending the minimum luminosity, i.e. the least massive structures, to which X-ray clusters can be detected and analyzed at intermediate redshifts. Galaxy groups and clusters with $k T \lesssim 4 \mathrm{keV}$ are starting to be routinely detected and analyzed in detail at $0.2<z<0.6$ (Willis et al. 2005; Gaga et al. 2005; Jeltema et al. 2006; Puccetti et al. 2006), where few examples were known. They represent the population which Chandra and in particular XMM surveys (like the $X M M$-LSS, Pierre et al. 2004) are sampling using typical exposures (10-20 ks), as expected (Jones et al. 2002).

These objects are more likely to display the effects of non-gravitational energy into the intra-cluster medium (ICM) than hotter more massive clusters (e.g., Ponman et al. 2003). The study of X-ray extended objects over an extended temperature range at $z>0.2$ will provide an important insight into the evolution of their $\mathrm{X}$-ray emitting gas and the deviation of X-ray scaling relations from simple, self-similar expectations. Studies of objects in the redshift range $0.2<z<0.6$ and with 2 $\mathrm{keV}<T<2.6 \mathrm{keV}$ are already suggesting that at these redshift these objects are less dynamically evolved that their counterparts at $z=0$ (Mulchaey et al. 2006). Fur-

\footnotetext{
${ }^{1}$ Department of Physics and Astronomy, University of California at Irvine, 4129 Frederick Reines Hall, Irvine, CA 92697-4575

2 Dipartimento di Astronomia, Università di Bologna, via Ranzani 1 , Bologna 40127, Italy

3 Dipartimento di Fisica, Università di Roma "La Sapienza", P.le A. Moro 2, Rome 00185, Italy

${ }^{4}$ Dipartimento di Fisica, Università di Roma "Tor Vergata", via della Ricerca Scientifica 1, Rome 00133, Italy

${ }^{5}$ Istituto di Astrofisica Spaziale e Fisica Cosmica (IASF/Roma), INAF, Rome, Italy
}

thermore, clusters with masses in the range $3-5 \times 10^{14}$ $\mathrm{M}_{\odot}\left(3 \mathrm{keV}<\mathrm{T}_{\mathrm{X}}<4 \mathrm{keV}\right)$ will constitute the bulk of the cosmological constraining power of future SZ surveys, because this is the range well-above the nearly redshift independent detection limit of these surveys (Haiman et al. 2001), therefore constituting the largest population in number count studies.

Here we present details of the $X M M$ observation of the cluster Zw 1305.4+2941, also known as MS 1305+29 with $k T \sim 3 \mathrm{keV}$ at the redshift of $z=0.241$, observed during an exposure of the field SA57. All distance-dependent quantities have been computed assuming $H_{0}=70 \mathrm{~km} \mathrm{~s}^{-1}$ $\mathrm{Mpc}^{-1}, \Omega_{\mathrm{m}}=0.3$ and $\Omega_{\Lambda}=0.7$. At the redshift of $z=$ $0.2411^{\prime}$ corresponds to $228 \mathrm{kpc}$. All the errors quoted are at the $68 \%$ confidence limit.

\section{X-RAY ANALYSIS}

The object $\mathrm{Zw}_{\mathrm{w}} 1305.4+2941$ has been observed during an $X M M$ pointing of the field SA 57 (Trevese et al. 2007) and it is located at $9.3^{\prime}$ off-axis (see Fig.1 ${ }^{6}$. The data were reduced with SAS v7.0.0 using the tasks emchain and epchain. We considered only event patterns 0-12 for MOS and 0 for pn and the data were cleaned using the standard procedures for bright pixels and hot columns removal (by applying the expression FLAG $==0$ ) and pn out-of-time correction. Periods of high backgrounds due to soft protons were filtered as in Gastaldello et al. (2007a); the observation was affected by flares at the end of the exposure and $\sim 20 \mathrm{ks}$ were lost, resulting in a net exposure time of 47,48 and 40 ks respectively for MOS1, MOS2 and pn.

For each detector we created images in the $0.5-2$ $\mathrm{keV}$ band with point sources, detected using the task ewavelet, masked using circular regions of $25^{\prime \prime}$ radius centered at the source position. The images have been exposure corrected and a radial surface brightness profile was extracted from a circular region of $6^{\prime}$ of radius centered

\footnotetext{
6 The center of the pointing has been calculated as an effectivearea-weighted average of the optical axis of the three telescopes taken form the exposure map headers keywords XCEN and YCEN, as in Pacaud et al. (2006)
} 


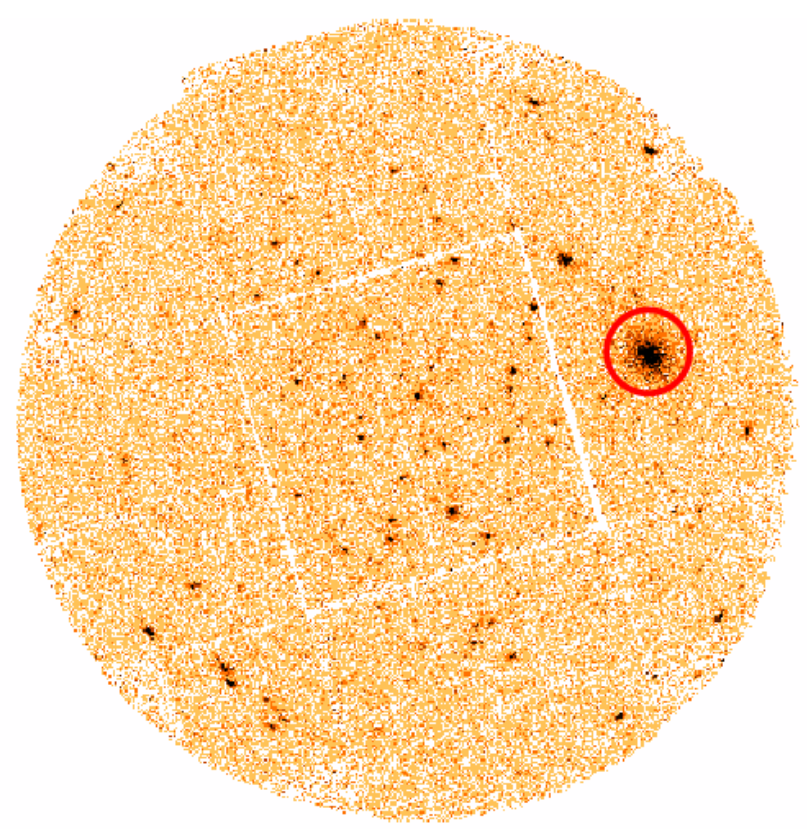

FIG. 1. - Exposure corrected 0.5-2.0 keV combined MOS1 and MOS 2 X-ray image of the field SA 57. The cluster Zw $1305.4+2941$ is clearly visible as the extended source and it is highlighted by the red circle of $1.5^{\prime}$ radius.

on the cluster centroid. We account for the X-ray background by including a constant-background component. The data were grouped to have at least 20 counts per bin in order to apply the $\chi^{2}$ statistic. The fitted model is convolved with the XMM PSF. The joint best-fit $\beta$-model (Cavaliere \& Fusco-Femiano 1976) has a core radius of $r_{c}=57 \pm 8 \mathrm{kpc}\left(14.9^{\prime \prime} \pm 2.0^{\prime \prime}\right)$ and $\beta=0.54 \pm 0.02$ for a $\chi^{2} /$ d.o.f. $=202 / 130$ (see Fig 2). Fits to the profiles of the individual detectors give consistent results within $1 \sigma$ of the combined-fit result and in the case of the MOS detectors are formally acceptable (20/24 MOS 1 and 41/39 MOS 2). The main contribution to the $\chi^{2}$ comes mainly from the pn and its origin is instrumental, hence there is no need for more complicated models.

For spectral fitting, we extracted spectra for each detector from a $1^{\prime}$ region centered on the centroid of the emission, to maximize the $\mathrm{S} / \mathrm{N}$ over the background. Redistribution matrix files (RMFs) and ancillary response files (ARFs) were generated using the SAS tasks rmfgen and arfgen in extended source mode. Appropriate flux-weighting was performed for RMFs and for ARFs, using exposure-corrected images of the source as detector maps (with pixel size of $1^{\prime}$, the minimum scale modeled by arfgen) to sample the variation in emission, following the prescription of Saxton \& Siddiqui (2002). The background was estimated locally using spectra extracted from a $2^{\prime}-3^{\prime}$ annular region centered on the centroid of the emission. The spectra from the three detectors were re-binned to ensure a signal-to-noise ratio of at least 3 and a minimum 20 counts per bin and they were jointly fitted with with an APEC thermal plasma modified by Galactic absorption (Dickey \& Lockman 1990). The spectral fitting was performed with Xspec (ver11.3.1, Arnaud 1996) in the 0.5-6 keV band and quoted metallicities are relative to the abundances of Grevesse \& Sauval (1998). The spectra are shown in Fig 3 the best fit parameters are $k T=3.17 \pm 0.19 \mathrm{keV}$ and $Z=0.93_{-0.29}^{+0.24}$
$Z_{\odot}$ for a $\chi^{2} /$ d.o.f. $=241 / 222$.

Using the best-fit model, the unabsorbed flux within the aperture of radius $1^{\prime}(228 \mathrm{kpc})$ is $2.07 \pm 0.06 \times 10^{-13}$ ergs $\mathrm{cm}^{-2} \mathrm{~s}^{-1}$ in the $0.5-2 \mathrm{keV}$ band. This corresponds to an unabsorbed luminosity of $3.33 \pm 0.15 \times 10^{43} \mathrm{ergs} \mathrm{s}^{-1}$ in the $0.5-2 \mathrm{keV}$ and to a bolometric $(0.01-100 \mathrm{keV})$ of $8.86 \pm 0.98 \times 10^{43} \mathrm{ergs} \mathrm{s}^{-1}$. The quoted errors on flux and luminosity are the mean and standard deviation of the distributions evaluated repeating the measurements after 10000 random selection of temperature, metallicity and normalization, drawn from Gaussian distributions with mean and standard deviation in accordance to the best fit results.

To investigate possible spatial variation in the spectral parameters of the cluster, we extracted two annular regions of radii $0^{\prime}-0.5^{\prime}$ and $0.5^{\prime}-1.5^{\prime}$. The derived spectral parameters are: $k T=3.04 \pm 0.23 \mathrm{keV}$ and $Z=0.93 \pm 0.45$ $Z \odot$ with $\chi^{2} /$ d.o.f. $=140 / 121$ for the inner annulus; $k T=3.47_{-0.22}^{+0.22} \mathrm{keV}$ and $Z=0.91_{-0.25}^{+0.32} Z_{\odot}$ with $\chi^{2} /$ d.o.f. $=153 / 159$ for the outer annulus. The width of the bins have been chosen in order to avoid bias in the temperature measurement caused by scattered flux by the PSF ( $80 \%$ encircled energy fraction radius is $31^{\prime \prime}$ for the pn at $1.5 \mathrm{keV}$ and at the off-axis angle of the source). The cluster is therefore consistent with being isothermal over the explored radial range.

The cluster has regular X-ray isophotes and is centered on a dominant early type galaxy (see 43 ). These characteristics suggest the cluster is relaxed and that hydrostatic equilibrium is a good approximation. The isothermal profile is not exceptional in a relaxed cluster, as Zw $1305.4+2941$ seems to have properties very similar to the low redshift cluster A 2589 (Zappacosta et al. 2006). We calculated the total mass profile using two different models. First, we used the best-fit $\beta$-model for which the gas density and total mass profiles can be expressed by simple analytical formula (e.g., Ettori 2000). 


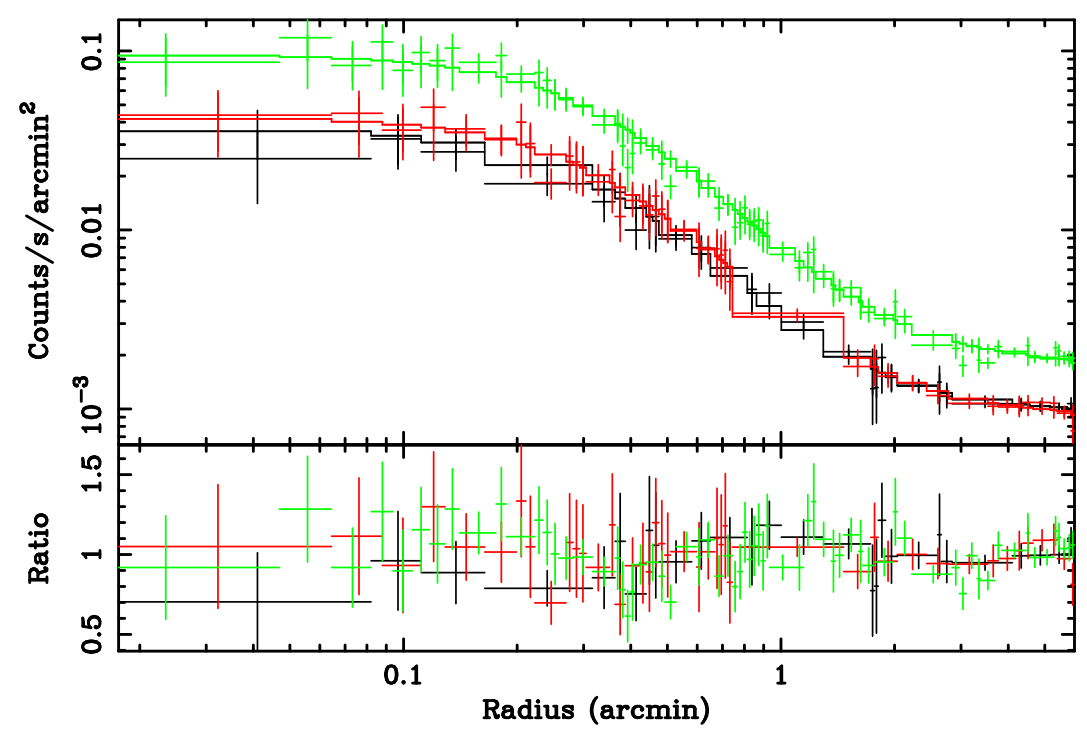

FIG. 2.- Surface brightness profile of the X-ray emission of Zw 1305.4+2941. Data from MOS1, MOS2 and pn are plotted in black, red and green respectively. The best fit beta model and ratio of data over the model are also shown.

EPIC 1 arcmin aperture

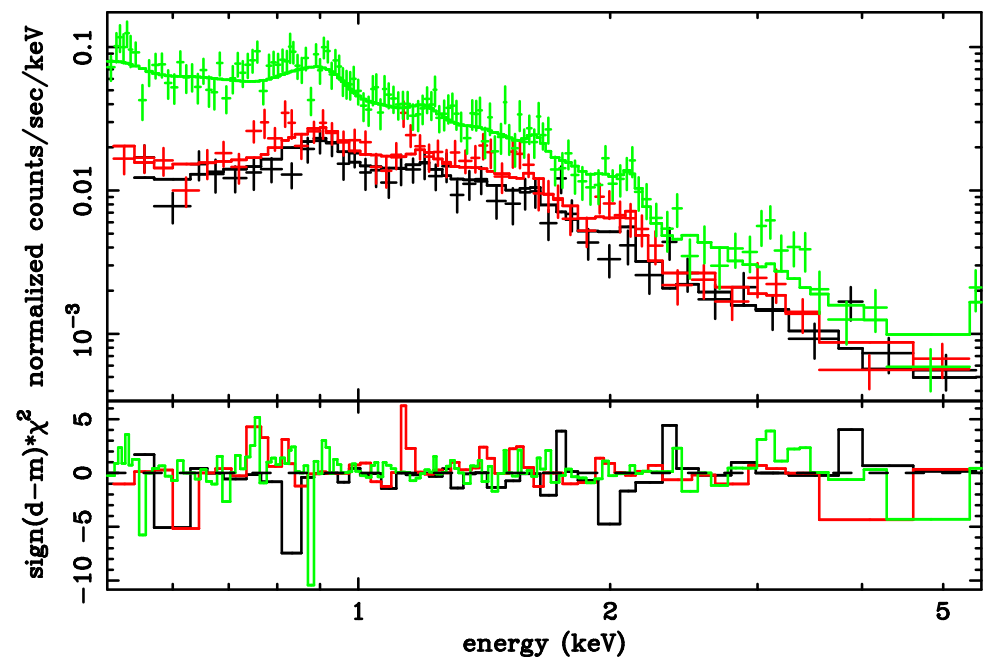

FIG. 3.- X-ray spectrum of the $\mathrm{Zw} 1305.4+2941$ taken from a $1^{\prime}$ aperture centred on the centroid of the emission. Data from MOS1, MOS2 and pn are plotted in black, red and green respectively. The best fit model and residuals are also shown.

We evaluated $r_{500}$ as the radius at which the density is 500 times the the critical density and the virial radius as the radius at which the density corresponds to $\Delta_{\text {vir }}$, as obtained by Bryan \& Norman (1998) ${ }^{7}$ for the concordance cosmological model used in this paper. To evaluate the errors on the estimated quantities, we repeat the measurements after 10000 random selections of a temperature and parameters of the surface brightness profile, which were drawn from Gaussian distributions with mean and variance in accordance with the best-fit results. For $\Delta=500$ we obtained, $M_{500}=(1.37 \pm 0.15) \times 10^{14}$ $\mathrm{M}_{\odot}$ within $r_{500}=723 \pm 16 \mathrm{kpc}$; the virial mass is, $M_{\text {vir }}=(2.81 \pm 0.30) \times 10^{14} \mathrm{M}_{\odot}$, within the virial radius $r_{\mathrm{vir}}=1474 \pm 52 \mathrm{kpc}$. Secondly, we fit the surface bright-

\footnotetext{
${ }^{7} \Delta_{v i r}=18 \pi^{2}+82 x-39 x^{2}$ where $x=\Omega(z)-1, \Omega(z)=\Omega_{0}(1+$ $z)^{3} / E(z)^{2}$ and $E(z)=\left[\Omega_{m}(1+z)^{3}+\Lambda\right]^{1 / 2}$
}

ness profile with an isothermal NFW (Navarro et al. 1997) model (Suto et al. 1998). We obtain a concentration parameter, $c=7.9 \pm 0.5$, virial radius $r_{\mathrm{vir}}=1468_{-41}^{+34}$ $\mathrm{kpc}$, and virial mass $M_{\text {vir }}=(2.77 \pm 0.21) \times 10^{14} \mathrm{M}_{\odot}$, with $\chi^{2} /$ d.o.f. $=197 / 130$. The mass determinations using the two different models agree well within the $1 \sigma$ errors. We calculate the gas mass using the procedure described in Ettori et al. (2004), using the $\beta$-model parametrization and deriving the central electron density from a combination of the surface brightness fit and the normalization of the spectral model (equation (2) of Ettori et al. 2004). We obtained $M_{\text {gas }, 500}=(1.37 \pm 0.14) \times 10^{13}$ $\mathrm{M}_{\odot}$. We calculate the entropy of the cluster using the standard definition $S=T_{g a s} / n_{e}^{2 / 3}$ and measure this quantity at $0.1 r_{200}$ and $r_{500}$ as done by Ponman et al. (2003). We find $S\left(0.1 r_{200}\right)=168 \pm 12 \mathrm{keV} \mathrm{cm}{ }^{2}$ and 
$S\left(r_{500}\right)=1103 \pm 122 \mathrm{keV} \mathrm{cm}{ }^{2}$, being $r_{200}=1144 \pm 41$ kpc using the best-fit $\beta$-model.

Finally, we have studied the sensitivity of our spectral results to various sources of systematic errors which we summarize below.

Galactic Column Density and Bandwidth: If $N_{\mathrm{H}}$ is allowed to vary the fit in the $1^{\prime}$ aperture does not improve and the best fit column density is consistent at $1 \sigma$ with the Galactic value; the other parameters are unchanged. Restricting the energy band to the $0.5-5 \mathrm{keV}$ band returned practically unchanged values, $k T=3.15 \pm 0.23$ and $Z=0.91 \pm 0.29$, whereas using a $0.4-5 \mathrm{keV}$ band has the effect of slightly increase the values, $k T=3.24 \pm 0.12$ and $Z=1.06_{-0.12}^{+0.34}$, but still with systematic errors less than the statistical ones.

Background: For comparison with the results obtained with the local background we used the standard blank background fields (Read \& Ponman 2003), finding good agreement between the two methods in the $1^{\prime}$ aperture, $k T=3.20 \pm 0.12$ and $Z=0.87 \pm 0.23$. In the $0.5^{\prime}$ $1.5^{\prime}$ outer annulus the results obtained with the background template are in agreement within $1 \sigma$ with the local background method, $k T=3.41_{-0.19}^{+0.25}$ and $Z=$ $0.70_{-0.19}^{+0.22}$.

Plasma Code: We investigated the sensitivity of our results to the plasma code using the MEKAL model. The quality of the fit and the temperature and abundance values were found to be very consistent within the $1 \sigma$ errors, $k T=3.24 \pm 0.13$ and $Z=0.89_{-0.10}^{+0.18}$.

\section{OPTICAL ANALYSIS}

Zw 1305.4+2941 is a Bautz-Morgan type I cluster at $\mathrm{Z}=0.24$. The optical map with over-plotted X-ray contours from $X M M 0.5-10 \mathrm{keV}$ data is shown in Fig 4 Optical photometry of the field SA 57 was obtained from $\mathrm{U}, B_{J}, \mathrm{~F}, \mathrm{~N}$ plates taken at KPNO $4 \mathrm{~m}$ Mayall telescope (Koo et al. 1986). A foreground cluster was recognized in the two-colour diagram calibrated with the spectroscopic redshifts available in the field (Koo et al. 1988). The cluster is elongated, with a major axis position angle $\theta_{c}=57^{\circ} \pm 6^{\circ}$. The central $\mathrm{cD}$ galaxy has a position angle $\theta_{c D}=61^{\circ}$ and an axial-ratio $b / a=0.78$, both measured at 3.5 magnitudes fainter than the central surface brightness and with $\mathrm{a} \approx 10 \%$ error. In Koo et al. (1988) the galaxy number density profiles, as deduced respectively in circular or elliptical annuli and statistically corrected for background, were fitted with both a projected Emden isothermal profile $\sigma(b)=\sigma_{o} F_{\text {isot }}\left(b / b_{c}\right)$ and projected King profile $\sigma_{a}(b)=\sigma_{a_{0}}\left[1+\left(b / R_{c}\right)^{2}\right]^{-1}$ following the procedure of Sarazin (1980). The parameters are the core radius $R_{c}=3 b_{c}=3 \sigma_{r} /\left(4 \pi G n_{0} m\right)^{1 / 2}$, the central surface density and the surface density of the background; $\sigma_{r}$ is the dispersion of the radial velocities, $n_{0}$ the central value of the volume density of galaxies and $m$ is the average galaxy mass. The results of the fit are reported in Table $2 \mathrm{~B}$ of Koo et al. (1986).

Mahdavi \& Geller (2001) report a velocity dispersion value $\sigma_{r}$ of $\sim 813 \mathrm{~km} \mathrm{~s}^{-1}$ citing the paper of Wu et al. (1999), but this value is not attributed in unequivocal way to the cluster MS 1305.4+2941. Mushotzky \& Scharf (1997) used velocity dispersion data from Fadda et al. (1996), Carlberg et al. (1996) and Fabricant et al. (1991), none of which contains $\sigma_{r}$ for MS
1305.4+2941. On the basis of the redshifts found using the Nasa Extragalactic Database $(N E D)$, mostly derived by the spectroscopic survey of the field by Munn et al. (1997), we have determined the velocity dispersion of the cluster considering circular areas of increasing radius, centered on the $\mathrm{cD}$ galaxy. The results are shown in Fig 5. where the redshifts as a function of the angular distance from the cluster center are also reported. The velocity dispersion is roughly constant between 1 and $3.5^{\prime}$ and progressively rises beyond $4^{\prime}$. This may be due to the inclusion of galaxies not belonging to the cluster or to incomplete virialization. The X-ray surface brightness falls below $3 \%$ of the central value at a radius of $3.3^{\prime}$ (see Fig 2), which corresponds to about $r_{500}$, and becomes practically undetectable. Therefore we adopt $\sigma\left(3.3^{\prime}\right)=568 \pm 125 \mathrm{~km} \mathrm{~s}^{-1}$ (based on 10 members) which corresponds to $\beta_{\text {spec }} \equiv \mu m_{p} \sigma_{r}^{2} / k T=0.64 \pm 0.31$ for $\mathrm{kT}$ $=3.17 \mathrm{keV}$ and $\mu=0.6$. This value of $\beta_{\text {spec }}$ is not much different from the value of $\beta_{\text {fit }}$ derived from the X-ray brightness distribution ( $0.54 \pm 0.02)$. Moreover, $\beta_{\text {spec }}<1$ is also consistent with the X-ray image that in the central region seems to show a quite relaxed cluster. A value of $\beta_{\text {spec }}$ lower than 1 may be due to the transfer from orbital to internal energy occurring in galaxy merging, thus cooling the galaxy velocity distribution despite the counteracting effect of the cluster gravitational potential (Fusco-Femiano \& Menci 1995). Finally, the fraction of blue galaxies $f_{b} \sim 0.1$ derived within $1.4^{\prime}$ from Koo et al. (1988, see their Fig.8) for this cluster is consistent with the relationship between cluster velocity dispersion and blue fraction within $r_{200} / 4$ obtained by Andreon et al. (2006), although not definitely conclusive.

\section{DISCUSSION}

In this section we discuss the properties of Zw $1305.4+2941$ in relation to the ones of objects with $k T \lesssim 4 \mathrm{keV}$ at the intermediate redshift $0.2<z<0.6$.

In particular we investigate the scaling relations between $L_{x}, T_{X}$ and velocity dispersion $\sigma_{v}$ for the six objects at intermediate redshift $(0.29<z<0.44)$ in the $X M M$ LSS survey (Willis et al. 2005), the six objects $(0.23<z<0.59)$ in the sample of Jeltema et al. (2006) and $\mathrm{Z}_{\mathrm{w}} 1305.4+2941$ together with the recently discovered cluster XMMUJ 131359.7-162735, with $k T=$ $3.57 \pm 0.12$, presented in Gastaldello et al. (2007b). We made a first attempt at investigating quantitatively these relations (in a simple power-law representation) in its normalization and slope by performing a linear regression $\log _{10} Y=\alpha \log _{10} X+b$ between two sets of measured quantities $Y$ and $X$. We employ the bisector modification of the BCES method (i.e., bivariate correlated errors with intrinsic scatter) described by Akritas \& Bershady (1996) that takes into account both any intrinsic scatter and errors on the two variables considered as symmetric. We performed the BCES fitting using software kindly provided by M. Bershady ${ }^{8}$. The uncertainties on the best fit results are obtained from $10^{5}$ bootstrap re-sampling. The results on the best-fit normalization and slope for the scaling laws here investigated are quoted in Table 1. together with total and intrinsic scatter (measured using equation (2) of Buote et al. 2007) and they are shown as black solid lines in Fig 6 .

\footnotetext{
8 http://www.astro.wisc.edu/ mab/archive/stats/stats.html
} 


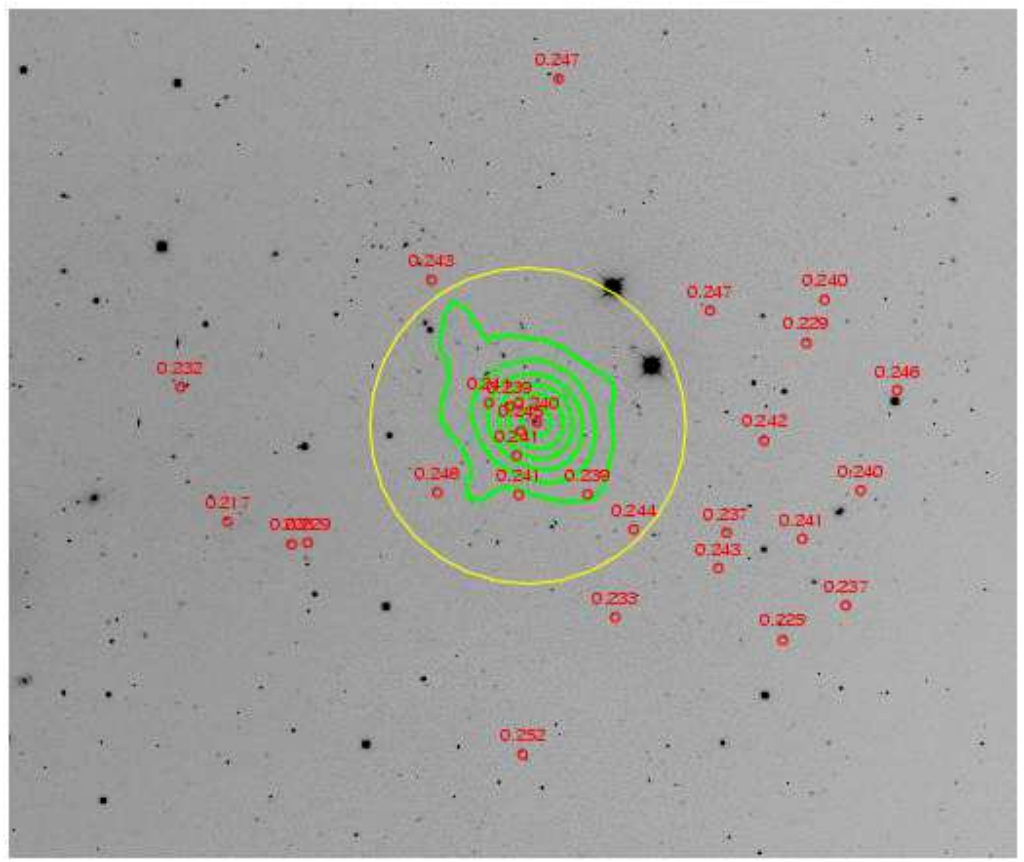

FIG. 4.- Optical map of the cluster ZwCl 1305.4+2941 with over-plotted X-ray contours from XMM-Newton 0.5-10 keV data. Galaxies in the redshift interval $0.21<z<0.27$ are indicated. The yellow circle marks a region with radius $3^{\prime}$.

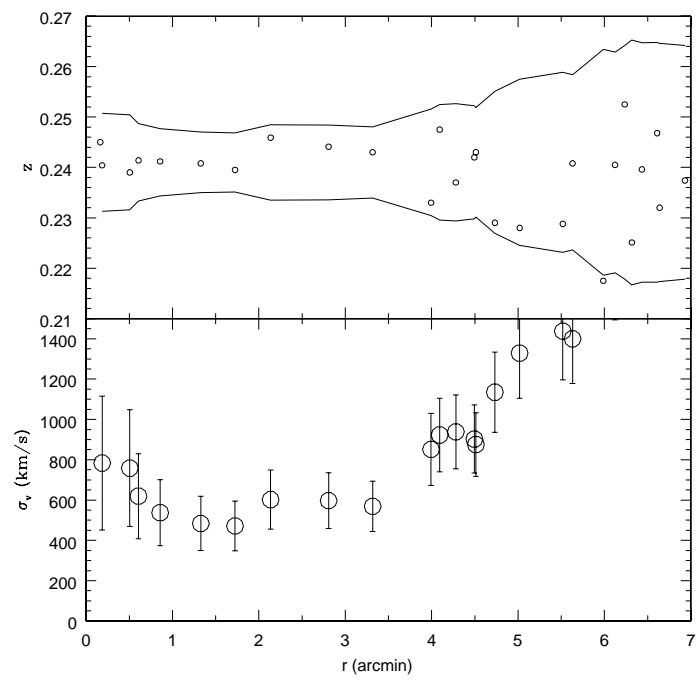

FIG. 5.- Upper panel: redshifts of galaxies in the area of $\mathrm{ZwCl}$ $1305.4+2941$ as a function of the distance from the cluster center. The lines represent $3 \sigma$ limits around the cluster redshift. Lower panel: Velocity dispersion as a function of the distance from the cluster center. Each error bar represent the r.m.s. velocity dispersion computed by randomly extracting, $10^{4}$ times, $N_{\text {gal }}(<r)$ velocities from gaussian distributions with $\sigma_{v}(<r)$, where $N_{\text {gal }}(<r)$ and $\sigma_{v}(<r)$ are the number and velocity dispersion of galaxies within distance $r$ from the cluster center.

We first investigate the $L_{X}-T_{X}$ relation. For $\mathrm{Zw}_{\mathrm{w}} 1305.4+2941$, the aperture of $228 \mathrm{kpc}$ used for spectroscopy encloses $70 \%$ of the flux within $r_{500}$, assuming the cluster emission profile follows the $\beta$-model of Fig.2 The derived bolometric luminosity within $r_{500}$ is $L_{500}=(1.26 \pm 0.16) \times 10^{44} \mathrm{ergs} \mathrm{s}^{-1}$; errors in the luminosity were determined including both the spectral errors and the uncertainties in the $\beta$-model parameters. The temperature derived with $X M M$ is in good agreement with the previous ROSAT determination $\left(2.98_{-0.41}^{+0.52} \mathrm{keV}\right.$, $2 \sigma$ errors, Mushotzky \& Scharf 1997). In the first panel of Fig 6 we plot the results for the intermediate redshift groups/poor clusters compared to the best fit regression lines for the low redshift groups of the GEMS sample (Osmond \& Ponman 2004), for the clusters of Horner (2001) as quoted in Osmond \& Ponman (2004), removing cool $\left(T_{X}<2 \mathrm{keV}\right)$ low luminosity $\left(L_{X}<2 \times 10^{43}\right.$ ergs $\mathrm{s}^{-1}$ ) objects and for the cluster sample of objects with $T_{X}>3 \mathrm{keV}$ of Markevitch (1998). With the caveat of the large error bars in the measured slope due to the still rather large errors in both luminosity and temperature and the small size of the sample, the relation is consistent with what found for local clusters. Given the angular resolution of the data it has not been possible to correct for the effect of central cool cores, which tend to reduce the scatter and produce flatter slopes (Allen \& Fabian 1998; Markevitch 1998).

We then investigate the relationship between the velocity dispersion of the group member galaxies and the X-ray temperature, excluding from the sample of Willis et al. (2005) XLSSC 013 for which no velocity dispersion was quoted and XMMUJ 131359.7-162735 for which we do not have optical spectroscopy. For the sample of Jeltema et al. (2006) we used the updated velocity dispersions presented in Jeltema et al. (2007). In the second panel of Fig 6 we show the $\sigma_{v}-T_{X}$ relation compared to the best fit of the GEMS groups (Osmond \& Ponman 2004), the cluster data of Horner (2001) as quoted in Osmond \& Ponman (2004) and the cluster sample of Girardi et al. (1996). As discussed in Jeltema et al. (2006) there is a large scatter with few groups which appears to have significantly low velocity dispersions for their temperature, similar to what found in the GEMS sample at lower X-ray luminosities and temperatures. A well known observational effect could be a possible explanation, due to the fact that velocity 

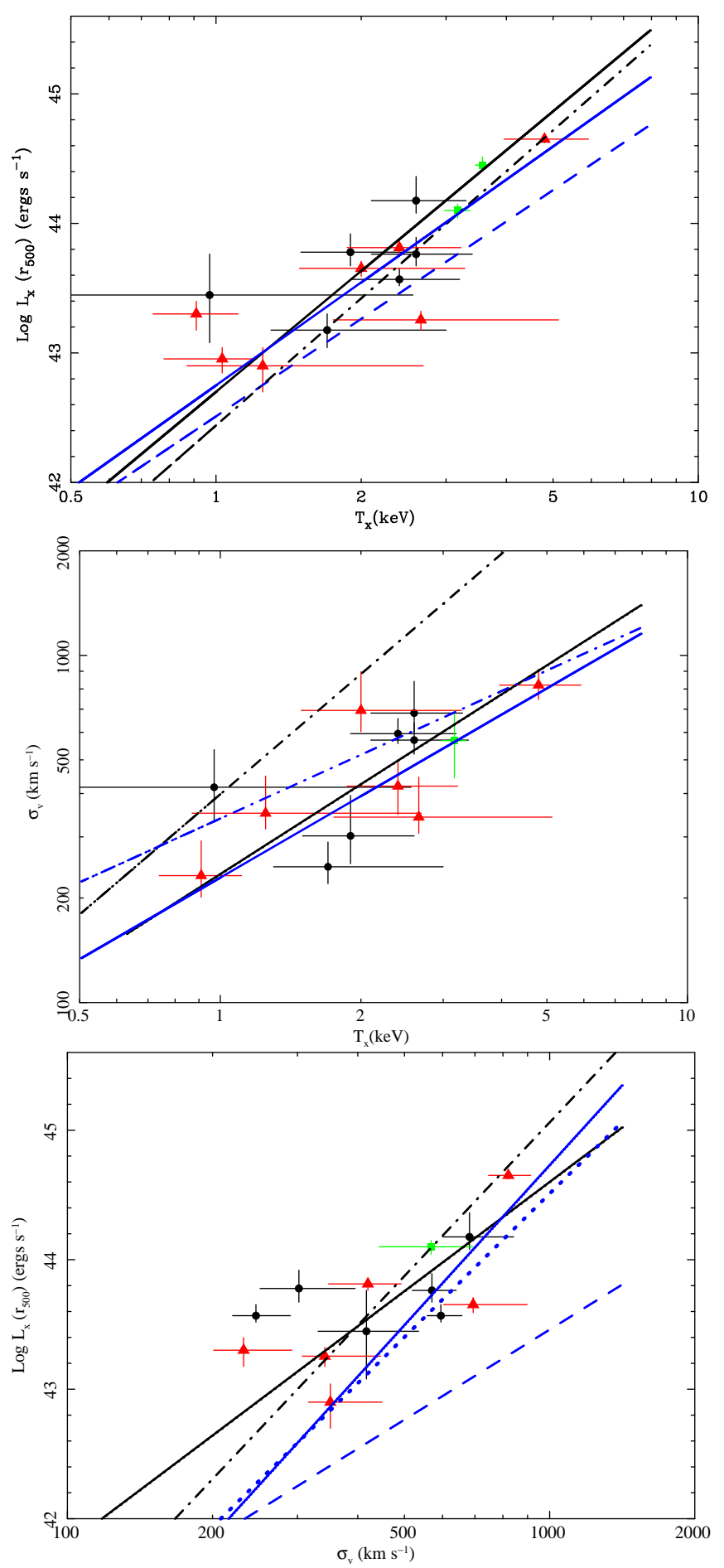

FIG. 6.- Top panel: $L_{X}-T_{X}$ relation for $\mathrm{Zw}_{\mathrm{w}} 1305.4+2941$ and the object discussed in Gastaldello et al. (2007b) (green squares), the moderate redshift sample of Willis et al. (2005) (red triangles) and the one of Jeltema et al. (2006)(black circles). The solid black line represents the best fit, the dot-dashed black line the fit to the cluster sample of Horner (2001) as discussed in Osmond \& Ponman (2004), the solid blue line the fit to the clusters of Markevitch (1998) and the blue dashed line the fit to the groups of the GEM sample (Osmond \& Ponman 2004).

Central panel: $\sigma_{v}-T_{X}$ relation with the best fit (black solid line). Also shown are the best fits to the cluster sample of Horner (2001) (solid blue line) and Girardi et al. (1996) (dot-dashed blue line). The dot-dashed black line shows the best fit to the GEMS group sample.

Lower panel: $L_{X}-\sigma_{v}$ relation with the best fit (black solid line). Also shown are the best fits to the cluster sample of Horner (2001) (dash-dotted black line), the REFLEX subsample of Ortiz-Gil et al. (2004) (solid blue line) and the RASS-SDSS sample of Popesso et al. (2005) (dotted blue line). The dashed blue line shows the best fit to the GEMS group sample. dispersions may be artificially low when based on relatively small numbers (e.g., Zabludoff \& Mulchaev 1998; Girardi \& Mezzetti 2001). Or velocity dispersions could be really reduced: Helsdon et al. (2005) propose several possible mechanisms for this effect, including dynamical friction, tidal heating and orientation effect (see also discussion in 93 ). The former explanation holds for many of the objects in the sample of Jeltema et al. (2006): for example RX J1334.9+3750 increased its velocity dispersion from $121_{-45}^{+58} \mathrm{~km} \mathrm{~s}^{-1}$ based on 6 members (Mulchaey et al. 2006) to $246_{-26}^{+44} \mathrm{~km} \mathrm{~s}^{-1}$ based on 17 members (Jeltema et al. 2007); RX J1648.7+6019 increased its velocity dispersion from $130_{-48}^{+46} \mathrm{~km} \mathrm{~s}^{-1}$ based on 8 members to $417_{-86}^{+118} \mathrm{~km} \mathrm{~s}^{-1}$ based on 22 members. Deeper spectroscopy to increase the robustness of the determination of the velocity dispersion and deeper X-ray observations are therefore crucial to clarify the nature of these systems. In the cluster regime there seems to be a consensus for a slight departure from a pure gravitational collapse, $\sigma_{v} \propto T^{\approx 0.6}$ (e.g., Lubin \& Bahcall 1993; Bird et al. 1995; Girardi et al. 1996; Xue \& Wu 2000), whereas the evidence for groups is more controversial, with authors (e.g., Mulchaev 2000; Xue \& Wu 2000) finding that groups fall on the cluster trend and others (Helsdon \& Ponman 2000b a) finding that the relation steepens. As discussed in Osmond \& Ponman (2004) the large non-statistical scatter mentioned above contributes to the controversy. Again with the caveat of large error bars, the objects in the intermediate-redshift sample seem to indicate an intermediate slope between clusters and low-temperature groups (but see Ortiz-Gil et al. 2004, for an even steeper slope, $1.00 \pm 0.16$, for a large REFLEX cluster sample). It is interesting to quote the results we would obtain had we used the velocity dispersions based on fewer members for the sample of Jeltema et al. (2006) reported in Mulchaey et al. (2006): a slope of $0.89 \pm 1.18$ and intercept $2.30 \pm 0.18$.

Finally we investigate the $L_{X}-\sigma_{v}$ relation for the same objects considered above, shown in the third panel of Fig.6. The cluster relation slope is consistently found by many investigations close to the purely gravitational expectation of 4 (e.g. Girardi \& Mezzetti 2001; Popesso et al. 2005; Ortiz-Gil et al. 2004, see the latter reference for a thorough comparison with previous determinations). There is disagreement at the group scale between studies which find that groups are consistent with the cluster relation (e.g., Helsdon \& Ponman 2000b; Mahdavi \& Geller 2001) and those which find significantly flatter relations (e.g., Xue \& Wu 2000; Osmond \& Ponman 2004). The results from the intermediate redshift sample point to a flatter trend compared to the cluster results. But we can see that this is mainly due to the same low velocity dispersion objects which have not only $T_{X}$ but also $L_{X}$ higher compared to the expectations. Clearly these are not the X-ray underluminous optically selected objects found both at the cluster (e.g., Popesso et al. 2007) and group scale (e.g., Rasmussen et al. 2006) believed to be systems which are collapsing for the first time and it is therefore likely that a better determination of $\sigma_{v}$ in these X-ray selected objects will bring these objects in closer agreement to the cluster scaling relations (modulo the effects described for example in Helsdon et al. 2005). In fact, for exam- 
ple, had we used the velocity dispersions based on fewer members reported in Mulchaev et al. (2006), we would have obtained a flatter slope of $2.07 \pm 1.21$ and intercept $38.3 \pm 3.2$.

We checked that the values for slope and intercept of the regression lines do not depend on the choice of the pivot point: we fitted the relation $\log _{10} Y / Y_{0}=$ $\alpha^{\prime} \log _{10} X / X_{0}+b^{\prime}$ with $L_{0}=10^{43.7} \mathrm{ergs} \mathrm{s}^{-1}, T_{0}=2.0$ $\mathrm{keV}$ and $\sigma_{0}=300 \mathrm{~km} / \mathrm{s}$, at the center of the data point cloud, in the three relations obtaining identical results to the ones in Table1.

\section{TABLE 1}

BEST FIT RESULTS ON THE SCALING RELATIONS.

\begin{tabular}{ccccc}
\hline Relation $(Y-X)$ & $\alpha$ & $b$ & $\sigma_{Y}$ & $\sigma_{Y}^{\text {intr }}$ \\
\hline$L_{X}-T_{X}$ & $3.10 \pm 1.77$ & $42.7 \pm 0.6$ & 0.39 & 0.37 \\
$\sigma_{v}-T_{X}$ & $0.86 \pm 0.85$ & $2.37 \pm 0.27$ & 0.14 & 0.12 \\
$L_{X}-\sigma_{v}$ & $2.82 \pm 0.75$ & $36.2 \pm 2.0$ & 0.36 & 0.33 \\
\hline
\end{tabular}

Note. - Best fit results for the scaling relations discussed in the text. The total scatter $\sigma_{Y}$ on $Y$ is measured as $\sigma_{Y}=$ $\left[\sum_{j=1, N}\left(\log Y_{j}-\alpha-A \log X_{j}\right)^{2} / N\right]^{1 / 2}$. The intrinsic scatter is estimated as $\sigma_{Y}^{\text {intr }}=\left(\sigma_{Y}^{2}-\sigma_{Y, \text { stat }}^{2}\right)^{1 / 2}$ where $\sigma_{Y, \text { stat }}^{2}=$ $\sum_{j=1, N}\left(1 / \sigma_{y_{j}}^{2}\right) / N$.

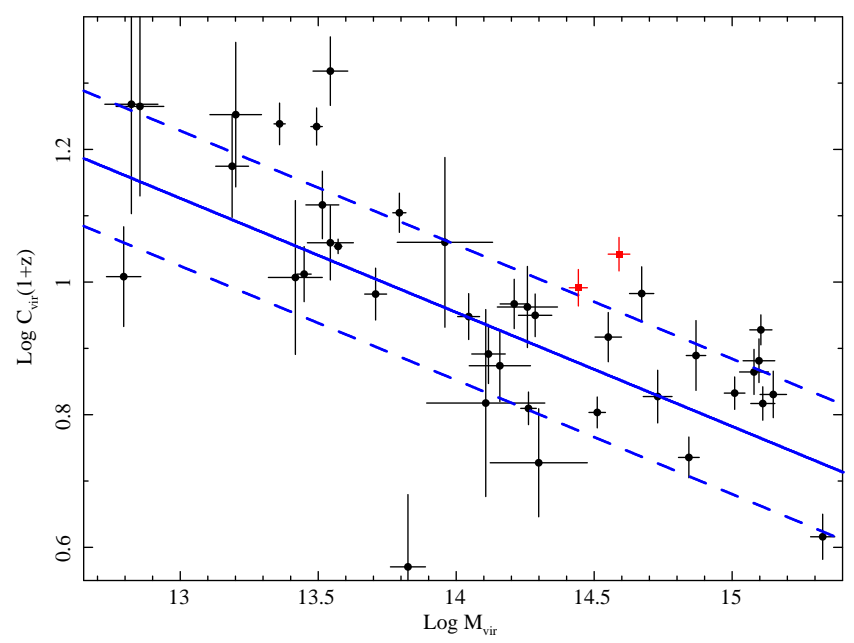

FIG. 7.- Local $c-M$ relation with data points (black circles) and best-fitting model (solid blue line) with $1 \sigma$ intrinsic scatter (dashed blue lines) as discussed in Buote et al. (2007). Over-plotted are the results for $\mathrm{Zw} 1305.4+2941$ and XMMUJ 131359.7-162735 with red squares.

The entropy for $\mathrm{Zw} 1305.4+2941$, measured at the two different over-density of $0.1 r_{200}$ and $r_{500}$ is consistent with the excess entropy observed in low redshift groups: scaling our results as $E_{z}^{4 / 3} S$ with $E_{z}=H_{z} / H_{0}=$ $\left[\Omega_{m}(1+z)^{3}+\Lambda\right]^{1 / 2}$ to account for the variation of the mean density within a given over-density radius with redshift, we find $E_{z}^{4 / 3} S\left(0.1 r_{200}\right)=232 \pm 16 \mathrm{keV} \mathrm{cm}{ }^{2}$ and $E_{z}^{4 / 3} S\left(r_{500}\right)=1522 \pm 168 \mathrm{keV} \mathrm{cm}{ }^{2}$ which can be compared with the plots in Fig.12 and Fig.13 of Jeltema et al. (2006) showing good agreement with the local relation
The high iron abundance for this cluster is consistent with the trend of increasing metallicity with decreasing temperature found at intermediate and high redshift by Balestra et al. (2007) with Chandra and XMM data and with the sample of Baumgartner et al. (2005) with $A S C A$ data. Particularly relevant is the comparison with objects like $\mathrm{Zw}_{\mathrm{w}} 0024.0+1652$ and V1416+4446 in the sample of Balestra et al. (2007) which at $\mathrm{z}=0.395$ and $\mathrm{z}=0.400$ have $k T=4.38 \pm 0.27, Z=1.09_{-0.26}^{+0.28}$ and $k T=3.50 \pm 0.18, Z=1.34_{-0.24}^{+0.31}$ respectively, having converted their abundances from Asplund et al. (2005) to Grevesse \& Sauval (1998) by scaling for 0.89. Also these objects, observed with Chandra and for which an analysis in two annuli is performed, do not show a clear enhancement of the iron abundance in the inner regions (Balestra et al. 2007), displaying the same behavior as $\mathrm{Zw}_{\mathrm{w}} 1305.4+2941$. These data seem to suggest therefore that the higher abundance is not due to the presence of a particularly iron-rich cool core. Zw $1305.4+2941$ is present in the sample of Baumgartner et al. (2005) based on the results of Horner (2001): the ASCA abundance determination is high $\left(0.92_{-0.59}^{+0.86}, 90 \%\right.$ errors, having converted their abundances from Anders \& Grevesse (1989) to Grevesse \& Sauval (1998) by scaling for 1.48) and in good agreement with the $X M M$ measurement. This trend between metallicity and temperature, still poorly understood, needs to be investigated with better data (it should be remarked that the constraints on the metallicity of $\mathrm{Zw} 1305.4+2941$ are interesting but not tight: it is consistent with $\sim 0.3 Z_{\odot}$ at the $2 \sigma$ level).

The measured concentration parameter $c$, multiplied by the expected dependence $1+z$ (Bullock et al. 2001) is consistent with a relaxed, early forming object in a $\Lambda$ CDM model with $\sigma_{8}=0.9$ and with the observational results of relaxed, low- $z$ objects (Buote et al. 2007). In Fig 7 we show the data points corresponding to Zw 1305.4+2941 and XMMUJ 131359.7-162735 overplotted to the $c-M$ data points and best-fit relation discussed in Buote et al. (2007). It should be kept in mind that these two data points have been derived under a very simple and restrictive isothermal assumption, whereas all the low- $z$ data points have been derived with a detailed investigation of the density, temperature and abundance profiles.

\section{CONCLUSIONS}

We present results for an $X M M$ observation of the cluster $\mathrm{Zw} 1305.4+2941$, for which we derive $k T=3.17 \pm$ $0.19 \mathrm{keV}$, an abundance of $0.93_{-0.29}^{+0.24} Z_{\odot}$ for an unabsorbed bolometric luminosity of $8.86 \pm 0.98 \times 10^{43} \mathrm{ergs} \mathrm{s}^{-1}$ within an aperture of $1^{\prime}(228 \mathrm{kpc}$ at $z=0.241)$. Under the assumption of isothermality and that the cluster follows the best-fit model to the surface brightness profile, we derive luminosity, entropy and mass at various over-densities. We measure a velocity dispersion of $568 \pm 125 \mathrm{~km} \mathrm{~s}^{-1}$ within $3.3^{\prime}$. These interesting constraints increase the small number of well studied $\sim 3$ keV objects above $z=0.1$.

We provide new fits of scaling relations for all literature $k T \lesssim 4 \mathrm{keV}$ objects in the intermediate- $z$ range. The cluster obeys the scaling relations thus derived. Concentration and mass for this object agree with the local $c-M$ relation. 
The prospects for increasing the sample size and improving the description presented here are promising. $X M M$ and Chandra are dramatically expanding our previous little knowledge of $\mathrm{X}$-ray emitting low-temperature clusters and groups of galaxies beyond the present epoch. Surveys like $X M M$-LSS (Pierre et al. 2004) and COSMOS (Finoguenov et al. 2007) will provide large samples of X-ray selected groups and poor clusters out to redshift of $z \sim 0.6$ or higher. Together with very large redshift surveys, optically selected groups in large quantities at moderate redshifts (e.g., Wilman et al. 2005b,a) will be obtained, characterizing in great detail this population and investigating some initial suggestion of group downsizing. More massive groups could be still in the process of virializing at intermediate redshift, while this process is restricted to much less luminous (and thus less massive) systems at present day (Mulchaev et al. 2006). X-ray follow-up with Chandra could allow to go beyond the simple isothermal beta model used so far in these studies.

We would like to thank D.A. Buote and S. Ettori for useful discussions. P.J. Humphrey is thanked for the use of his surface brightness fitting code. Partial support for this work was provided by the ASI/INAF grants n. I/023/05/0 and n. I/088/06/0. This work is based on observations obtained with $X M M$ an ESA science mission with instruments and contributions directly funded by ESA member states and the USA (NASA). This research has made use of data obtained from the High Energy Astrophysics Science Archive Research Center (HEASARC), provided by NASA's Goddard Space Flight Center. This research has also made use of the NASA/IPAC Extragalactic Database (NED) which is operated by the Jet Propulsion Laboratory, California Institute of Technology, under contract with NASA.
Akritas, M. G. \& Bershady, M. A. 1996, ApJ, 470, 706

Allen, S. W. \& Fabian, A. C. 1998, MNRAS, 297, L57

Anders, E. \& Grevesse, N. 1989, Geochim. Cosmochim. Acta, 53, 197

Andreon, S., Quintana, H., Tajer, M., Galaz, G., \& Surdej, J. 2006, MNRAS, 365, 915

Arnaud, K. A. 1996, in ASP Conf. Ser. 101: Astronomical Data Analysis Software and Systems V, Vol. 5, 17

Asplund, M., Grevesse, N., \& Sauval, A. J. 2005, in Astronomical Society of the Pacific Conference Series, Vol. 336, Cosmic Abundances as Records of Stellar Evolution and Nucleosynthesis, ed. T. G. Barnes, III \& F. N. Bash, 25-+

Balestra, I., Tozzi, P., Ettori, S., Rosati, P., Borgani, S., Mainieri, V., Norman, C., \& Viola, M. 2007, A\&A, 462, 429

Baumgartner, W. H., Loewenstein, M., Horner, D. J., \& Mushotzky, R. F. 2005, ApJ, 620, 680

Bird, C. M., Mushotzky, R. F., \& Metzler, C. A. 1995, ApJ, 453, 40

Bremer, M. N., Valtchanov, I., Willis, J., Altieri, B., Andreon, S. Duc, P. A., Fang, F., Jean, C., Lonsdale, C., Pacaud, F. Pierre, M., Shupe, D. L., Surace, J. A., \& Waddington, I. 2006, MNRAS, 371, 1427

Bryan, G. L. \& Norman, M. L. 1998, ApJ, 495, 80

Bullock, J. S., Kolatt, T. S., Sigad, Y., Somerville, R. S., Kravtsov, A. V., Klypin, A. A., Primack, J. R., \& Dekel, A. 2001, MNRAS, 321, 559

Buote, D. A., Gastaldello, F., Humphrey, P. J., Zappacosta, L., Bullock, J. S., Brighenti, F., \& Mathews, W. G. 2007, ApJ, 664,123

Carlberg, R. G., Yee, H. K. C., Ellingson, E., Abraham, R., Gravel, P., Morris, S., \& Pritchet, C. J. 1996, ApJ, 462, 32

Cavaliere, A. \& Fusco-Femiano, R. 1976, A\&A, 49, 137

Dickey, J. M. \& Lockman, F. J. 1990, ARA\&A, 28, 215

Ettori, S. 2000, MNRAS, 311, 313

Ettori, S., Tozzi, P., Borgani, S., \& Rosati, P. 2004, A\&A, 417, 13

Fabricant, D. G., McClintock, J. E., \& Bautz, M. W. 1991, ApJ, 381,33

Fadda, D., Girardi, M., Giuricin, G., Mardirossian, F., \& Mezzetti, M. 1996, ApJ, 473, 670

Finoguenov, A., Guzzo, L., Hasinger, G., Scoville, N. Z., Aussel,

H., Böhringer, H., Brusa, M., Capak, P., Cappelluti, N., Comastri, A., Giodini, S., Griffiths, R. E., Impey, C.

Koekemoer, A. M., Kneib, J.-P., Leauthaud, A., Le Fèvre, O., Lilly, S., Mainieri, V., Massey, R., McCracken, H. J., Mobasher B., Murayama, T., Peacock, J. A., Sakelliou, I., Schinnerer, E., Silverman, J. D., Smolčić, V., Taniguchi, Y., Tasca, L., Taylor, J. E., Trump, J. R., \& Zamorani, G. 2007, ApJS, 172, 182 Fusco-Femiano, R. \& Menci, N. 1995, ApJ, 449, 431

Gaga, T., Plionis, M., Basilakos, S., Georgantopoulos, I., \& Georgakakis, A. 2005, MNRAS, 363, 811

\section{REFERENCES}

Gastaldello, F., Buote, D. A., Humphrey, P. J., Zappacosta, L., Bullock, J. S., Brighenti, F., \& Mathews, W. G. 2007a, ApJ, 669,158

Gastaldello, F., Buote, D. A., Humphrey, P. J., Zappacosta, L., Seigar, M. S., Barth, A. J., Brighenti, F., \& Mathews, W. G. 2007b, ApJ, 662, 923

Girardi, M., Fadda, D., Giuricin, G., Mardirossian, F., Mezzetti, M., \& Biviano, A. 1996, ApJ, 457, 61

Girardi, M. \& Mezzetti, M. 2001, ApJ, 548, 79

Grevesse, N. \& Sauval, A. J. 1998, Space Science Reviews, 85, 161

Haiman, Z., Mohr, J. J., \& Holder, G. P. 2001, ApJ, 553, 545

Helsdon, S. F. \& Ponman, T. J. 2000a, MNRAS, 319, 933

- 2000b, MNRAS, 315, 356

Helsdon, S. F., Ponman, T. J., \& Mulchaey, J. S. 2005, ApJ, 618, 679

Horner, D. J. 2001, PhD thesis, University of Maryland

Jeltema, T. E., Mulchaey, J. S., Lubin, L. M., \& Fassnacht, C. D. 2007, ApJ, 658, 865

Jeltema, T. E., Mulchaey, J. S., Lubin, L. M., Rosati, P., \& Böhringer, H. 2006, ApJ, 649, 649

Jones, L. R., McHardy, I., Newsam, A., \& Mason, K. 2002, MNRAS, 334, 219

Koo, D. C., Kron, R. G., Nanni, D., Trevese, D., \& Vignato, A. 1986, AJ, 91, 478

Koo, D. C., Kron, R. G., Nanni, D., Vignato, A., \& Trevese, D. 1988, ApJ, 333, 586

Lubin, L. M. \& Bahcall, N. A. 1993, ApJ, 415, L17

Mahdavi, A. \& Geller, M. J. 2001, ApJ, 554, L129

Markevitch, M. 1998, ApJ, 504, 27

Mulchaey, J. S. 2000, ARA\&A, 38, 289

Mulchaey, J. S., Lubin, L. M., Fassnacht, C., Rosati, P., \& Jeltema, T. E. 2006, ApJ, 646, 133

Mullis, C. R., Rosati, P., Lamer, G., Böhringer , H., Schwope, A., Schuecker, P., \& Fassbender, R. 2005, ApJ, 623, L85

Munn, J. A., Koo, D. C., Kron, R. G., Majewski, S. R., Bershady, M. A., \& Smetanka, J. J. 1997, ApJS, 109, 45

Mushotzky, R. F. \& Scharf, C. A. 1997, ApJ, 482, L13+

Navarro, J. F., Frenk, C. S., \& White, S. D. M. 1997, ApJ, 490, 493

Ortiz-Gil, A., Guzzo, L., Schuecker, P., Böhringer, H., \& Collins, C. A. 2004, MNRAS, 348, 325

Osmond, J. P. F. \& Ponman, T. J. 2004, MNRAS, 350, 1511

Pacaud, F., Pierre, M., Refregier, A., Gueguen, A., Starck, J.-L. Valtchanov, I., Read, A. M., Altieri, B., Chiappetti, L., Gandhi, P., Garcet, O., Gosset, E., Ponman, T. J., \& Surdej, J. 2006, MNRAS, 372, 578 
Pierre, M., Valtchanov, I., Altieri, B., Andreon, S., Bolzonella, M., B remer, M., Disseau, L., Dos Santos, S., Gandhi, P., Jean, C., Pacaud, F., Read, A., Refregier, A., Willis, J., Adami, C., Alloin, D., Birkinshaw, M., Chiappetti, L., Cohen, A., Detal, A. an d Duc, P.-A., Gosset, E., Hjorth, J., Jones, L., LeFevre, O., Lonsdale, C., Maccagni, D., Mazure, A., McBreen, B., McCracken, H., Mellier, Y., Ponman, T., Quintana, H., Rottgering, H., Smette, A., Surdej, J. an d Starck, J.-L., Vigroux, L., \& White, S. 2004, Journal of Cosmology and Astro-Particle Physics, 9, 11

Ponman, T. J., Sanderson, A. J. R., \& Finoguenov, A. 2003, MNRAS, 343, 331

Popesso, P., Biviano, A., Böhringer, H., \& Romaniello, M. 2007, A\&A, 461, 397

Popesso, P., Biviano, A., Böhringer, H., Romaniello, M., \& Voges, W. 2005, A\&A, 433, 431

Puccetti, S., Fiore, F., D'Elia, V., Pillitteri, I., Feruglio, C., Grazian, A., Brusa, M., Ciliegi, P., Comastri, A., Gruppioni, C., Mignoli, M., Vignali, C., Zamorani, G., La Franca, F., Sacchi, N., Franceschini, A., Berta, S., Buttery, H., \& Dias, J. E. 2006, A\&A, 457, 501

Rasmussen, J., Ponman, T. J., Mulchaey, J. S., Miles, T. A., \& Raychaudhury, S. 2006, MNRAS, 373, 653

Read, A. M. \& Ponman, T. J. 2003, A\&A, 409, 395

Sarazin, C. L. 1980, ApJ, 236, 75

Saxton, R. D. \& Siddiqui, H. 2002, XMM-SOC-PS-TN-43
Stanford, S. A., Romer, A. K., Sabirli, K., Davidso n, M., Hilton, M., Viana, P. T. P., Collins, C. A., Kay, S. T., Liddle, A. R., Mann, R. G., Miller, C. J. a nd Nichol, R. C., West, M. J., Conselice, C. J., Spinrad, H., Stern, D., \& Bundy, K. 2006, ApJ, 646, L13

Suto, Y., Sasaki, S., \& Makino, N. 1998, ApJ, 509, 544

Trevese, D., Vagnetti, F., Puccetti, S., Fiore, F., Tomei, M., \& Bershady, M. A. 2007, A\&A, 469, 1211

Willis, J. P., Pacaud, F., Valtchanov, I., Pierre, M., Ponman, T., Read, A., Andreon, S., Altieri, B., Quintana, H., Dos Santos, S., Birkinshaw, M., Bremer, M., Duc, P.-A., Galaz, G., Gosset, E., Jones, L., \& Surdej, J. 2005, MNRAS, 363, 675

Wilman, D. J., Balogh, M. L., Bower, R. G., Mulchaey, J. S., Oemler, A., Carlberg, R. G., Eke, V. R., Lewis, I., Morris, S. L., \& Whitaker, R. J. 2005a, MNRAS, 358, 88

Wilman, D. J., Balogh, M. L., Bower, R. G., Mulchaey, J. S., Oemler, A., Carlberg, R. G., Morris, S. L., \& Whitaker, R. J. 2005b, MNRAS, 358, 71

Wu, X.-P., Xue, Y.-J., \& Fang, L.-Z. 1999, ApJ, 524, 22

Xue, Y.-J. \& Wu, X.-P. 2000, ApJ, 538, 65

Zabludoff, A. I. \& Mulchaey, J. S. 1998, ApJ, 496, 39

Zappacosta, L., Buote, D. A., Gastaldello, F., Humphrey, P. J., Bullock, J., Brighenti, F., \& Mathews, W. 2006, ApJ, 650, 777 